\title{
A Simultaneous Optimization of Vehicle Routing when Picking and Delivering Multiple Products from Cross-Docks under Uncertainty Conditions
}

\author{
Mohammad Rahebi,Dr. Roya M.Ahari* \\ Department of Industrial Engineering, Najafabad Branch, Islamic Azad University, Najafabad, Iran
}

\begin{abstract}
Nowadays, it is essential to complete some operations as soon as possible in large distribution centers given the high number of customer visits. Regarding this, vehicle routing is so significant for both receiving raw materials from suppliers and sending demand to customer centers. Thus, it is essential to optimize vehicles routing simultaneously between supply and demand centers. Another significant point is using cross-docks. Moreover, considering vehicle moving times definite at the time of picking or delivering cargo can lead to poor quality responses that would lead to the delivery of the demand sooner or later to the customers than the determined deadline causing customer dissatisfaction with the logistic network of supplying demand. Thus, given the uncertainties in the time of transfer in cross-warehouses, the purpose of the study was to regulate the policies of receiving, sorting and labeling of multiple products for cross-docks as well as optimizing VRP routing of vehicles at two levels: 1) supply points and cross-docks and (2) cross-dock and delivery points to minimize overall operational costs. It is noteworthy that the time of vehicle movement was uncertain. Moreover, given the complexities of solving the stated problem, metaheuristic solution based on biogeography was presented and to examine the performance of the proposed solution method more, its results were compared with Particle Swarm Optimization (PSO) algorithm developed by Chen et al. (2016). To do so, the results of the scenarios were analyzed using PSO and Biogeography-Based Optimization (BBO) algorithms.
\end{abstract}

Keywords: Cross-docks, Vehicle routing, Uncertainty, Using scenarios

\section{Introduction}

One of the heuristic warehousing strategies with great ability in controlling distribution and logistics costs while maintaining the level of customer service is cross-dock. Nowadays, it is essential to complete operations such as receiving, sorting, labeling, and transferring goods as soon as possible in large distribution centers given the high number of customer visits. In such a situation, it is necessary to pay attention to two issues: the routing and the transfer between supply and demand centers by determining the structure of the movement of vehicles. Regarding this, vehicle routing is significant for both receiving raw materials from suppliers and sending the demands to customer centers. The purpose of Vehicle Routing Problem (VRP) is to minimize the total cost of transferring goods from the central station to customers (Dantzig and Ramsar, 1959). Vehicle routing has been very effective in the efficiency and effectiveness of transportation systems, supply chain and communications management, so it has attracted so much attention in recent years. Vehicle routing problems are significant for researchers from two aspects: one in obtaining an optimal response to a problem that leads to economical savings, and the other is that the presence of these types of problems in optimization problems is very difficult (Dehbari et al., 2012). Thus, it is necessary to optimize simultaneous vehicle routing between supply and demand centers. Another significant point is using cross-docks, so that the sorting and labeling machines are present. Cross-dock is a communication strategy in the new warehousing in the logistics system. Cross-dock is as an incorporator of products of the outgoing shipments, which can easily be sorted and categorized in a distribution center. For outgoing shipments, a distribution center is defined as a cross-dock in this case. This tool essentially eliminates the maintenance of inventories in the traditional warehouses of material classification and sends them to destinations (Apte and Viswanathan, 2000). Regarding this, products are taken from supplier nodes and shipped to cross-docks, and then, to estimate customers' demand, vehicles move towards delivery nodes (customers). Uncertainties in the external environment, like weather conditions, as well as traffic, render the vehicle's moving times to be unpredictable when picked up or delivered. Inattention to this issue can lead to poor quality responses that can deliver the demand early or later than the set time whose outcome is customer dissatisfaction with demand-supply logistics network. Regarding this, Arnaout et al. (2010) and Velasquez et al. (2010) introduced a simulation model for cross-dock network. They considered some probable parameters of delivery, demand, and service time and used a triangular distribution for order size and receipt dates. Barbucha (2012) solved the vehicle-routing problem with capacity limit using simultaneous meta-heuristic and 
cooperative methods, aimed at minimizing the number of vehicles. LiangjunKe and Zuren Feng (2013) proposed an heuristic method to solve the traffic routing problem with accumulated capacity limitation. Tas et al. (2013) examined the problem of vehicle routing and considered probable travel time and time window as flexiblein their studies. Their purpose was to optimize shipping and services. Javanmard et al. (2013) solved the problem of receiving and picking products, considering several products and time window. Moreover, they used cross-dock warehouse to reduce shipping costs. YosefiKhoshbakhat et al. (2014) proposed vehicle routing problem assuming open routes for airplanes. Goksal et al. (2013) examined a hybrid PSO method for VRP problem from two aspects of picking up and delivery of the product. In another study, Jafari et al. (2011) presented a hybrid simulated annealing (HSA) algorithm for solving a multi-warehouse vehicle routing problem. Ziaw et al. (2014) proposed a simulated annealing algorithm to solve CVRP problems. They used a combination of simulated local search algorithms and simulated annealing algorithms. In other words, they used VNS to search for the neighborhood structure in the SA algorithm. Gounaris et al. (2014) used the meta-heuristic algorithm of adaptive memory programming to solve the unsteady request CVRP problem. Madani-Isfahani et al. (2014) developed a complex integer programming model for the problem of timing of trucks in a multiplex cross-docks system to provide multiple meta-heuristic algorithms such as annealing simulation and firefly algorithms. Asadi and Bagheri (2016) studied the timing problem with the allocation of goods to minimize the number of delayed trucks. Lee et al. (2016) studied the timing of trucks assuming that interruptions in discharge operations were allowed in limited numbers. In this study, the goal was to minimize the scope of the operation. PSO algorithm has been used to solve the model at large-scale. Faiza Walha et al. (2016) defined cross-dock as a hybrid process that connected middle nodes to the transport network, aiming at reducing inventory storage and service time. In this paper, an overview of the cross-dock problem has been made despite the uncertainty. + ) They studied Tehran's fruit and vegetable distribution center. The center has cross-docks dams and limited time for load trucks. In this paper, it is assumed that the entry of the trucks into the dock with one gate is based on a uniform distribution in the interval (0.L). The number of trucks assigned and the loading time were constant. Amini and Tavakoli Moghaddam (2016) discussed the problem of scheduling trucks in a cross-dock, where the trucks may be in trouble during their service life. Indeed, the number of failures per time unit for each truck has Poisson's probability distribution. Briesemeister and Novaes (2017) have proposed an uncertain approximate model for determining the number of ditches that truck enters.

Given the stated points, th present study assumed the adjusting of the policies for the receipt, sorting and labeling of multiple products for cross-dock, as well as VRP optimization at two levels: one is the supply points and cross-docks, and the other cross-docks delivery points, so that the overall operating costs were minimized. Thus, the purpose of the study was simultaneous VRP optimization at picking up and delivery of multiple products from cross-docks under uncertainty conditions. 


\section{Mathematical modeling}

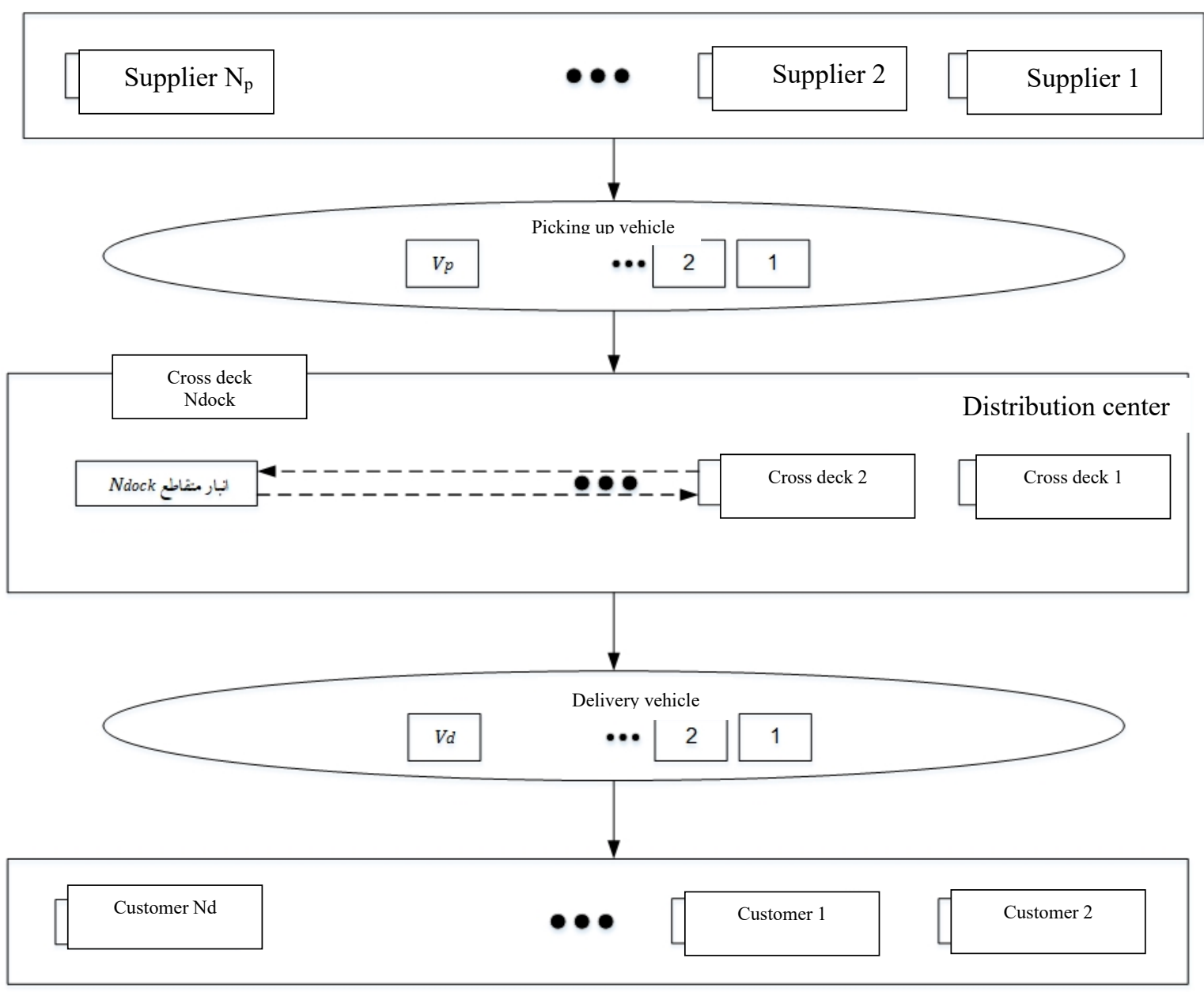

Figure 1. The structure of the research problem

\subsection{The set of parameters and variables of research problem}

The set of parameters and variables of the research problem is as follows:

Parameters

$\mathrm{N}_{\mathrm{p}}$ : The number of pick up nodes

$\mathrm{N}_{\mathrm{d}}$ : The number of delivery nodes

$\mathrm{C}_{\mathrm{d}}$ : Operating cost per dock

$\mathrm{C}_{\mathrm{h}}$ : The cost of each material review unit

$\mathrm{Ptc}_{\mathrm{ij}}$ : Transfer cost of a pickup truck from node $\mathrm{i}$ to node $\mathrm{j}$

Dtc $_{\mathrm{ij}}$ : Transfer cost of a delivery vehicle from node $\mathrm{i}$ to node $\mathrm{j}$

$\mathrm{V}_{\mathrm{p}}$ : Total number of pickup vehicles

$\mathrm{V}_{\mathrm{d}}$ : Total number of delivery vehicles

$\mathrm{Pc}_{\mathrm{k}}$ : Operating cost for the picking vehicle $\mathrm{k}$

$\mathrm{Dc}_{\mathrm{k}}$ : Operating cost for delivery vehicle $\mathrm{k}$

$\mathrm{P}_{\mathrm{i}}$ : The number of products collected from node $\mathrm{i}$

$D_{i j}$ : The number of $i$-type products evacuated from node $j$ (demanding product type $i$ for the delivery node $j$ ).

$\mathrm{u}_{\mathrm{ij}}$ : The number of transfers from node $\mathrm{i}$ to node $\mathrm{j}$ in pickup vehicle

$\mathrm{z}_{\mathrm{ij}}$ : The number of transfer from node $\mathrm{i}$ to node $\mathrm{j}$ in the delivery vehicle 
$\mathrm{Q}_{\mathrm{p}}$ : Maximum capacity of the vehicles

$\mathrm{Q}_{\mathrm{d}}$ : Maximum capacity of the delivery vehicles

$\mathrm{S}_{\mathrm{p}}$ : Sum of all pickup nodes

$\mathrm{S}_{\mathrm{d}}$ : Sum of all delivery nodes

$\mathrm{S}_{0}$ : The sum of all cross-docks

$\mathrm{TW}_{\text {pick: }}$ Time horizon for pick up

$\mathrm{TW}_{\text {del }}$ : Time horizon for delivery

$\mathrm{d}_{\mathrm{a}}$ : cross-dock a

$\mathrm{N}_{\text {dock}}$ : The number of cross-docks in the distribution center

$\mu t_{i j}^{k}$ : The mean time the vehicle moves from node $\mathrm{i}$ to node $\mathrm{j}$ with vehicle type $\mathrm{k}$

$\sigma t_{i j}^{k}$ : Standard deviation when the vehicle moves from node $\mathrm{i}$ to node $\mathrm{j}$ with vehicle type $\mathrm{k}$

$\mathrm{t}_{\mathrm{i}}$ : The time required for loading, discharging or cross-dock at node $\mathrm{i}$

\section{Decision variables}

$x_{i j}^{k}$ : If the pickup vehicle k moves from node $\mathrm{i}$ to node $\mathrm{j}$, it is 1 otherwise 0 .

$w_{i j}^{k}$ : If the k-th delivery vehicle moves from node $\mathrm{i}$ to node $\mathrm{j}$, it is equal to 1 otherwise 0 .

$V P_{d a}^{k}$ : If the k-th pickup vehicle is connected to da cross-dock, it is equal to 1 otherwise 0 .

$V D_{d a}^{k}$ : If the k-th delivery vehicle is connected to da cross-dock, it is equal to 1 otherwise 0 .

$q_{d_{a} d_{b}}^{i}$ : The value of the $\mathrm{i}$-th product moves from $\mathrm{d}_{\mathrm{a}}$ to $\mathrm{d}_{\mathrm{b}}$ cross-dock

\subsection{Mathematical model}

After expressing the variables and parameters, the proposed mathematical model of the research problem was as follows:

$$
\begin{gathered}
\operatorname{Min} \sum_{j=0}^{N_{p}} \sum_{i=0}^{N_{p}} \sum_{k=1}^{V_{p}} p t c_{i j} x_{i j}^{k}+\sum_{j=0}^{N_{d}} \sum_{i=0}^{N_{d}} \sum_{k=1}^{V_{d}} d t c_{i j} w_{i j}^{k}+\sum_{j=0}^{N_{p}} \sum_{k=1}^{V_{p}} p c_{k} x_{o j}^{k}+\sum_{j=0}^{N_{d}} \sum_{k=1}^{V_{d}} d c_{k} w_{o j}^{k} \\
+C_{h} \sum_{\substack{b=1 \\
N_{d o c h}}}^{N_{d o c k}} \sum_{a=1}^{V_{p}} \sum_{i=1}^{V_{p}=1} q_{d_{a} d_{b}}^{i}+C_{d} \sum_{k=1}^{N_{d o c k}} \sum_{a=1}^{N_{d o c k}} V P_{d_{a}^{k}} \sum_{j=1}^{N_{p}} x_{o j}^{k} \\
+C_{d} \sum_{k=1}^{N_{a=1}} \sum_{a=1}^{N_{d}} V D_{d_{a}^{k}} \sum_{j=1}^{k} x_{o j}^{k}
\end{gathered}
$$

s.t.

$$
\begin{aligned}
& \sum_{i=0}^{N_{p}} \sum_{k=1}^{N_{p}} x_{i j}^{k}=1 \quad \forall j \\
& \sum_{\substack{N_{p}=0 \\
V_{p}}}^{V_{k}} \sum_{i j}^{k}=1 \quad \forall j \\
& \sum_{\substack{N_{d} \\
V_{d}}}^{V_{d}} \sum_{k=1}^{N_{d}} w_{i j}^{k}=1 \quad \forall j \\
& \sum_{j=0}^{V_{d}} \sum_{k=1}^{N_{p}} w_{i j}^{k}=1 \quad \forall i \\
& \sum_{i=0}^{N_{p}} x_{i l}^{k}-\sum_{j=0}^{N_{p}} x_{l j}^{k}=0 \quad \forall l, k
\end{aligned}
$$




$$
\begin{aligned}
& \sum_{i=0}^{N_{d}} w_{i l}^{k}-\sum_{j=0}^{d} w_{l j}^{k}=0 \quad \forall l, k \\
& \sum_{k=0}^{V_{p}} \sum_{i=1}^{N_{p}} x_{0 i}^{k} \leq V_{p} \\
& \begin{array}{cc}
k=0 \\
V_{d} & N_{d} \\
&
\end{array} \\
& \sum_{k=1} \sum_{j=1}^{N_{d}} w_{0 j}^{k} \leq V_{d} \\
& u_{i j} \leq Q_{p} x_{i j}^{k} \quad \forall i, j, k \\
& z_{i j} \leq Q_{d} w_{i j}^{k} \quad \forall i, j, k \\
& \sum_{k=1}^{V_{p}} \sum_{j=0}^{N_{p}} V P_{d_{a}}^{k} P_{i} x_{i j}^{k}+\sum_{\substack{b=1 \\
b \neq a}}^{N_{\text {dock }}} q_{d_{b} d_{a}}^{i}-\sum_{\substack{b=1 \\
b \neq a}}^{N_{\text {dock }}} q_{d_{b} d_{a}}^{i}=\sum_{k=1}^{V_{d}} \sum_{j=0}^{N_{d}} \sum_{l=0}^{N_{d}} V D_{d_{a}}^{k} D_{i j} w_{i j}^{k} \quad \forall i, d_{a} \\
& u_{j l}-u_{i j}=\left\{\begin{array}{cc}
P_{j}^{b \neq a} & \text { for } j \in S_{p}^{b \neq a} \\
-\sum_{i=1}^{N_{p}} P_{i} & \text { for } j \in S_{0}
\end{array}\right. \\
& z_{i j}-z_{j l}=\left\{\begin{array}{cl}
\sum_{i=1}^{\text {allproducts }} D_{i j} & \text { for } j \in S_{d} \\
-\sum_{i=1}^{\text {allproducts }} \sum_{j=1}^{N_{d}} D_{i j} & \text { for } j \in S_{0}
\end{array}\right. \\
& \sum_{i=0}^{N_{p}} \sum_{j=0}^{N_{p}} t_{i}^{k} x_{i j}^{k}+\sum_{i=0}^{N_{p}} \sum_{j=0}^{N_{p}}\left(\mu t_{i j}^{k}+z_{\alpha} \sigma t_{i j}^{k}\right)+x_{i j}^{k} \leq T W_{\text {pick }} \quad \forall k \\
& \sum_{i=0}^{N_{d}} \sum_{j=0}^{N_{d}} t_{i}^{k} w_{i j}^{k}+\sum_{i=0}^{N_{d}} \sum_{j=0}^{N_{d}}\left(\mu t_{i j}^{k}+z_{\alpha} \sigma t_{i j}^{k}\right) w_{i j}^{k} \leq T W_{d e l} \quad \forall k \\
& \sum_{a=1}^{N_{\text {dock }}} V P_{d_{a}}^{k}=1 \quad \forall k \\
& \sum_{a=1}^{N_{\text {dock }}} V D_{d_{a}}^{k}=1 \quad \forall k \\
& x_{i j}^{k}, w_{i j}^{k}, V P_{d_{a}}^{k}, V D_{d_{b}}^{k} \in\{0,1\} \text { and } q_{d_{a} d_{b}}^{i} \geq 0
\end{aligned}
$$

The first four objective function showed the total transport costs associated with the pick-up and delivery vehicles. One has to note that the total cost of transportation includes shipping costs from suppliers and the cost of supplying goods to customers. In the objective function equation, the distribution center containing a crossdock warehouse is shown with o. The fifth sentence shows the cost of transporting goods between cross-dock warehouses. The sixth and seventh sentences show the total operating costs for collection and delivery in crossdock warehouses. The cost of transporting goods between cross-dock warehouses has been obtained from the product of the cost of each unit of delivery of goods $C_{h}$ in the quantity of products of type $i$ that need transportation $\left(q_{d_{a} d_{b}}^{i}\right)$.

Constraints (2) to (5) showed that only one vehicle (picking or delivery) can enter and exit the node. Constraints (6) and (7) showed the movement of return and delivery vehicles. Constraints (8) and (9) showed the notion that the number of vehicles taken and delivered in each cross-warehouse should be less than and equal to the total number of vehicles available. Constraints (10) and (11) showed that the number of goods loaded in a vehicle should not exceed the capacity of the pickup and delivery vehicles. Flow of products in the dock has been shown in the Constraint (12). The number of products in the route between the pickup and delivery nodes has been expressed in the Constraints (13) and (14). The total service time in each vehicle and the total transport time must be less than or equal to the pickup $\left(\mathrm{TW}_{\text {pick }}\right)$ and delivery $\left(\mathrm{TW}_{\text {del }}\right)$ time horizons. Constraints $(17)$ and (18) showed that each vehicle could only be assigned to one cross-dock warehouse. Constraint (19) showed the range of model variables. 


\subsection{Meta-heuristic solution method based on biogeography algorithm}

The structure of the answer to the research problem was a three-dimensional answer, under which the first level of VRP was related to suppliers, in the second level, the response of the customer-oriented VRP matrix and, finally, the third-dimensional matrix of the value of load sent from each product among cross-dock warehouses. Figure (2) shows the structure of the solution method of the proposed solution.

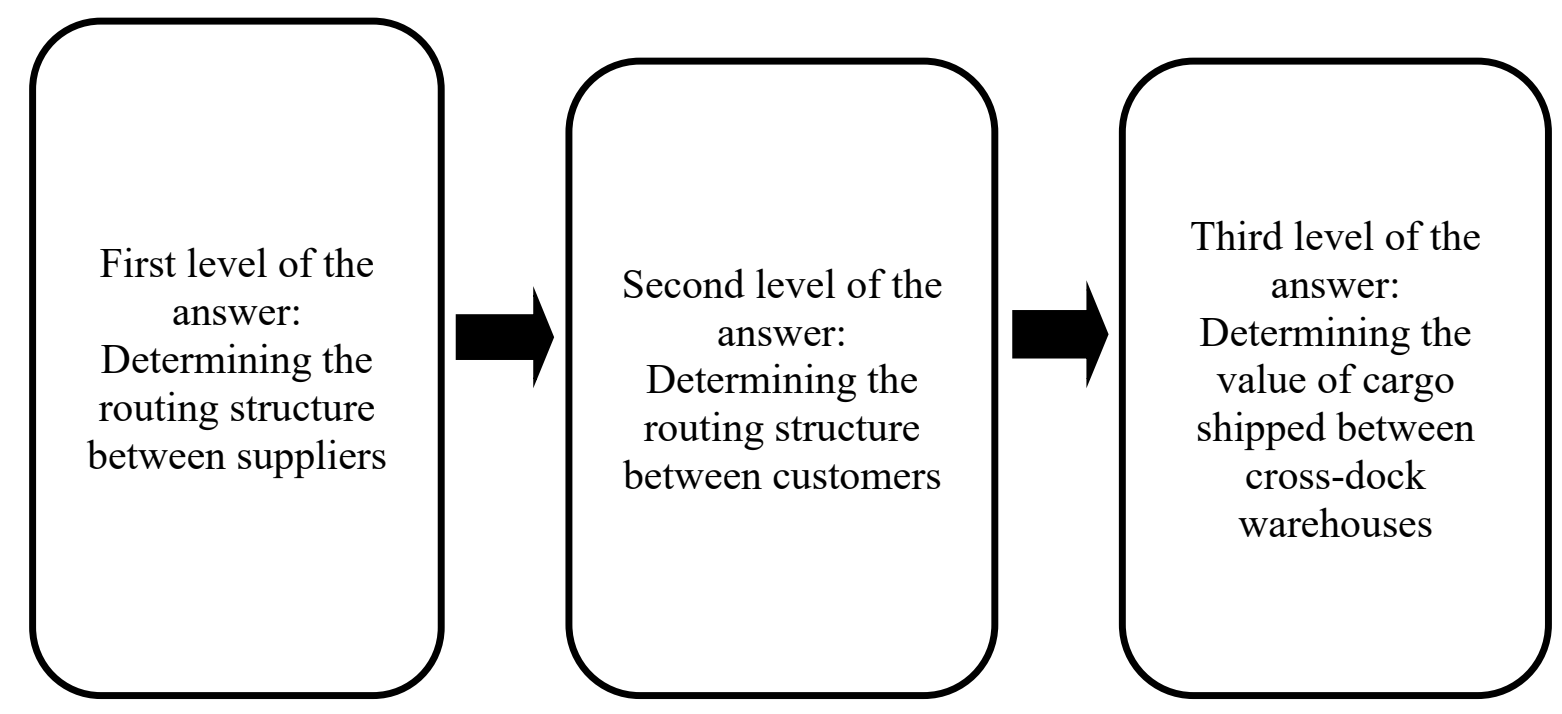

Figure 2. The structure of the answer of the proposed solution

To validate the proposed solution method, the mathematical model of the research problem was solved using GAMS software in small dimensions and the result has been compared with BBO algorithm. It should be noted that all calculations performed using the hardware system with specifications CPU G3250 $3.20 \mathrm{GHz} / \mathrm{RAM}$ 4GB and BBO algorithm coding in the software environment MTLAB R2016a. Overall, 64 scenarios were analyzed.

\section{Results}

In this section, numerical examples for the research problem have been soved. For this purpose, considering $N_{p} \in\{7,8,9,10\}, N_{d} \in\{7,8,9,10\}, Q_{p} \in\{3000,3500\}$ and $Q_{d} \in\{2000,3000\}, 64$ scenarios were analyzed.

In Table (1), numerical examples have been presented below. In these tables, column Np showed the number of suppliers. Column Nd represented the number of customers. Column Qp showed the capacity of the product transport vehicles between suppliers and the cross-dock and column Qd the capacity of the transport vehicle of the product between the cross-dock and the customers. Tour supplier column showed the optimal substrate obtained

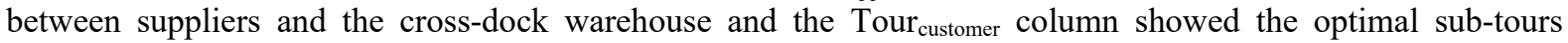
obtained between the cross-dock warehouse and customers. Column CPU (S) in GAMS part of the solving time was presented using GOM software and CPU column (S) in BBO section of the proposed solving algorithm solving time. Column $f$ showed theachieved value of objective function. Finally, in the Gap\% column, there was a gap between BBO and GAMS solutions. It should be noted that GOM software had only the ability to solve the problem in the dimensions of $\mathrm{Np} \in\{7\}$ and $\mathrm{Nd} \in\{7\}$ in less than 10,000 seconds, and for larger dimensions, practically accessing the optimal answer during this time given the hard problem was impossible. Thus, for larger dimensions, only solution results for BBO algorithm have been shown. 
Table 1. Results of optimization of the research problem using BBO algorithm

\begin{tabular}{|c|c|c|c|c|c|c|c|c|}
\hline \multirow{2}{*}{ 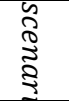 } & \multirow{2}{*}{$N_{p}$} & \multirow{2}{*}{$N_{d}$} & \multirow{2}{*}{$Q_{p}$} & \multirow{2}{*}{$Q_{d}$} & \multicolumn{4}{|c|}{$B B O$} \\
\hline & & & & & Tour $_{\text {supplier }}$ & Tour ${ }_{\text {customer }}$ & $f$ & $C P U(S)$ \\
\hline$S 1$ & 10 & 10 & 3500 & 3000 & $\begin{array}{r}57-49-29-17-57 \\
57-90-69-60-58-57 \\
57-88-98-99-57\end{array}$ & $\begin{array}{r}57-43-23-25-57 \\
57-64-52-55-57 \\
57-96-94-84-73-57\end{array}$ & 578451 & 124.8 \\
\hline$S 2$ & 10 & 10 & 3500 & 2000 & $\begin{array}{r}57-49-29-17-57 \\
57-90-69-60-58-57 \\
57-88-98-99-57\end{array}$ & $\begin{array}{r}57-43-23-25-57 \\
57-64-52-55-57 \\
57-84-73-57 \\
57-96-94-57\end{array}$ & 601589 & 123.5 \\
\hline$S 3$ & 10 & 10 & 3000 & 3000 & $\begin{array}{r}57-49-29-17-57 \\
57-69-60-58-57 \\
57-99-90-57 \\
57-88-98-57\end{array}$ & $\begin{array}{r}57-43-23-25-57 \\
57-64-52-55-57 \\
57-96-94-84-73-57\end{array}$ & 610844 & 124.0 \\
\hline$S 4$ & 10 & 10 & 3000 & 2000 & $\begin{array}{r}57-49-29-17-57 \\
57-69-60-58-57 \\
57-99-90-57 \\
57-88-98-57\end{array}$ & $\begin{array}{r}57-43-23-25-57 \\
57-64-52-55-57 \\
57-84-73-57 \\
57-96-94-57\end{array}$ & 663483 & 124.1 \\
\hline$S 5$ & 10 & 9 & 3500 & 3000 & $\begin{array}{r}57-49-29-17-57 \\
57-90-69-60-58-57 \\
57-88-98-99-57\end{array}$ & $\begin{array}{r}57-55-43-23-25-57 \\
57-96-94-84-73-64-57\end{array}$ & 464794 & 115.0 \\
\hline$S 6$ & 10 & 9 & 3500 & 2000 & $\begin{array}{r}57-49-29-17-57 \\
57-90-69-60-58-57 \\
57-88-98-99-57\end{array}$ & $\begin{array}{r}57-43-23-25-57 \\
57-84-73-64-55-57 \\
57-96-94-57\end{array}$ & 501048 & 114.8 \\
\hline$S 7$ & 10 & 9 & 3000 & 3000 & $\begin{array}{r}57-49-29-17-57 \\
57-69-60-58-57 \\
57-99-90-57 \\
57-88-98-57\end{array}$ & $\begin{array}{r}57-55-43-23-25-57 \\
57-96-94-84-73-64-57\end{array}$ & 547574 & 114.9 \\
\hline$S 8$ & 10 & 9 & 3000 & 2000 & $\begin{array}{r}57-49-29-17-57 \\
57-69-60-58-57 \\
57-99-90-57 \\
57-88-98-57\end{array}$ & $\begin{array}{r}57-43-23-25-57 \\
57-84-73-64-55-57 \\
57-96-94-57\end{array}$ & 553556 & 114.8 \\
\hline$S 9$ & 10 & 8 & 3500 & 3000 & $\begin{array}{r}57-49-29-17-57 \\
57-90-69-60-58-57 \\
57-88-98-99-57\end{array}$ & $\begin{array}{r}-84-64-73-43-23-25-57 \\
57 \\
57-96-94-57\end{array}$ & 402366 & 106.7 \\
\hline$S 10$ & 10 & 8 & 3500 & 2000 & $\begin{array}{r}57-49-29-17-57 \\
57-90-69-60-58-57 \\
57-88-98-99-57\end{array}$ & $\begin{array}{r}57-43-23-25-57 \\
57-84-73-64-57 \\
57-96-94-57 \\
\end{array}$ & 416328 & 106.9 \\
\hline$S 11$ & 10 & 8 & 3000 & 3000 & $\begin{array}{r}57-49-29-17-57 \\
57-69-60-58-57 \\
57-99-90-57 \\
57-88-98-57\end{array}$ & $\begin{array}{r}-84-64-73-43-23-25-57 \\
57 \\
57-96-94-57\end{array}$ & 457812 & 106.8 \\
\hline
\end{tabular}


Table 2. Results of optimization of the research problem using BBO algorithm (continued)

\begin{tabular}{|c|c|c|c|c|c|c|c|c|}
\hline \multirow{2}{*}{ 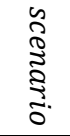 } & \multirow{2}{*}{$N_{p}$} & \multirow{2}{*}{$N_{d}$} & \multirow{2}{*}{$Q_{p}$} & \multirow{2}{*}{$Q_{d}$} & \multicolumn{4}{|c|}{$B B O$} \\
\hline & & & & & Tour ${ }_{\text {supplier }}$ & Tour $_{\text {customer }}$ & $f$ & $C P U(S)$ \\
\hline$S 12$ & 10 & 8 & 3000 & 2000 & $\begin{array}{r}57-49-29-17-57 \\
57-69-60-58-57 \\
57-99-90-57 \\
57-88-98-57\end{array}$ & $\begin{array}{r}57-43-23-25-57 \\
57-84-73-64-57 \\
57-96-94-57\end{array}$ & 517885 & 106.7 \\
\hline$S 13$ & 10 & 7 & 3500 & 3000 & $\begin{array}{r}57-49-29-17-57 \\
57-90-69-60-58-57 \\
57-88-98-99-57\end{array}$ & $\begin{array}{r}57-84-73-43-23-25-57 \\
57-96-94-57\end{array}$ & 304825 & 97.0 \\
\hline$S 14$ & 10 & 7 & 3500 & 2000 & $\begin{array}{r}57-49-29-17-57 \\
57-90-69-60-58-57 \\
57-88-98-99-57\end{array}$ & $\begin{array}{r}57-43-23-25-57 \\
57-94-84-73-57 \\
57-96-57\end{array}$ & 313299 & 97.1 \\
\hline$S 15$ & 10 & 7 & 3000 & 3000 & $\begin{array}{r}57-49-29-17-57 \\
57-69-60-58-57 \\
57-99-90-57 \\
57-88-98-57\end{array}$ & $\begin{array}{r}57-84-73-43-23-25-57 \\
57-96-94-57\end{array}$ & 339636 & 97.2 \\
\hline$S 16$ & 10 & 7 & 3000 & 2000 & $\begin{array}{r}57-49-29-17-57 \\
57-69-60-58-57 \\
57-99-90-57 \\
57-88-98-57\end{array}$ & $\begin{array}{r}57-43-23-25-57 \\
57-94-84-73-57 \\
57-96-57\end{array}$ & 380117 & 97.2 \\
\hline$S 17$ & 9 & 10 & 3500 & 3000 & $\begin{array}{r}57-58-49-29-17-57 \\
57-90-69-60-57 \\
57-88-99-57\end{array}$ & $\begin{array}{r}57-43-23-25-57 \\
57-64-52-55-57 \\
57-96-94-84-73-57\end{array}$ & 506470 & 115.1 \\
\hline$S 18$ & 9 & 10 & 3500 & 2000 & $\begin{array}{r}57-58-49-29-17-57 \\
57-90-69-60-57 \\
57-88-99-57\end{array}$ & $\begin{array}{r}57-43-23-25-57 \\
57-64-52-55-57 \\
57-84-73-57 \\
57-96-94-57\end{array}$ & 530443 & 115.2 \\
\hline$S 19$ & 9 & 10 & 3000 & 3000 & $\begin{array}{l}57-49-29-17-57 \\
57-69-60-58-57 \\
57-88-99-90-57\end{array}$ & $\begin{array}{r}57-43-23-25-57 \\
57-64-52-55-57 \\
57-96-94-84-73-57\end{array}$ & 588243 & 115.0 \\
\hline$S 20$ & 9 & 10 & 3000 & 2000 & $\begin{array}{l}57-49-29-17-57 \\
57-69-60-58-57 \\
57-88-99-90-57\end{array}$ & $\begin{array}{r}57-43-23-25-57 \\
57-64-52-55-57 \\
57-84-73-57 \\
57-96-94-57\end{array}$ & 603531 & 114.9 \\
\hline
\end{tabular}


Table 3. Results of optimization of the research problem using BBO algorithm

\begin{tabular}{|c|c|c|c|c|c|c|c|c|}
\hline \multirow{2}{*}{ 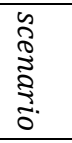 } & \multirow[b]{2}{*}{$N_{p}$} & \multirow[b]{2}{*}{$N_{d}$} & \multirow[b]{2}{*}{$Q_{p}$} & \multirow[b]{2}{*}{$Q_{d}$} & \multicolumn{4}{|c|}{$B B O$} \\
\hline & & & & & Tour $_{\text {supplier }}$ & Tour $_{\text {customer }}$ & $f$ & $C P U(S)$ \\
\hline$S 21$ & 9 & 9 & 3500 & 3000 & $\begin{array}{r}57-58-49-29-17-57 \\
57-90-69-60-57 \\
57-88-99-57\end{array}$ & $\begin{array}{r}57-55-43-23-25-57 \\
57-96-94-84-73-64-57\end{array}$ & 402663 & 100.2 \\
\hline$S 22$ & 9 & 9 & 3500 & 2000 & $\begin{array}{r}57-58-49-29-17-57 \\
57-90-69-60-57 \\
57-88-99-57\end{array}$ & $\begin{array}{r}57-43-23-25-57 \\
57-84-73-64-55-57 \\
57-96-94-57\end{array}$ & 428597 & 100.1 \\
\hline$S 23$ & 9 & 9 & 3000 & 3000 & $\begin{array}{l}57-49-29-17-57 \\
57-69-60-58-57 \\
57-88-99-90-57\end{array}$ & $\begin{array}{r}57-55-43-23-25-57 \\
57-96-94-84-73-64-57\end{array}$ & 449147 & 100.2 \\
\hline$S 24$ & 9 & 9 & 3000 & 2000 & $\begin{array}{l}57-49-29-17-57 \\
57-69-60-58-57 \\
57-88-99-90-57\end{array}$ & $\begin{array}{r}57-43-23-25-57 \\
57-84-73-64-55-57 \\
57-96-94-57\end{array}$ & 452314 & 100.1 \\
\hline$S 25$ & 9 & 8 & 3500 & 3000 & $\begin{array}{r}57-58-49-29-17-57 \\
57-90-69-60-57 \\
57-88-99-57\end{array}$ & $\begin{array}{r}-64-73-43-23-25-57 \\
57-84 \\
57-96-94-57\end{array}$ & 270589 & 90.8 \\
\hline$S 26$ & 9 & 8 & 3500 & 2000 & $\begin{array}{r}57-58-49-29-17-57 \\
57-90-69-60-57 \\
57-88-99-57\end{array}$ & $\begin{array}{r}57-43-23-25-57 \\
57-84-73-64-57 \\
57-96-94-57\end{array}$ & 309013 & 90.8 \\
\hline$S 27$ & 9 & 8 & 3000 & 3000 & $\begin{array}{l}57-49-29-17-57 \\
57-69-60-58-57 \\
57-88-99-90-57\end{array}$ & $\begin{array}{r}-64-73-43-23-25-57 \\
57-84 \\
57-96-94-57\end{array}$ & 335963 & 90.7 \\
\hline$S 28$ & 9 & 8 & 3000 & 2000 & $\begin{array}{l}57-49-29-17-57 \\
57-69-60-58-57 \\
57-88-99-90-57\end{array}$ & $\begin{array}{r}57-43-23-25-57 \\
57-84-73-64-57 \\
57-96-94-57\end{array}$ & 394248 & 91.0 \\
\hline$S 29$ & 9 & 7 & 3500 & 3000 & $\begin{array}{l}57-49-29-17-57 \\
57-69-60-58-57 \\
57-88-99-90-57\end{array}$ & $\begin{array}{r}57-84-73-43-23-25-57 \\
57-96-94-57\end{array}$ & 206242 & 86.7 \\
\hline$S 30$ & 9 & 7 & 3500 & 2000 & $\begin{array}{r}57-58-49-29-17-57 \\
57-90-69-60-57 \\
57-88-99-57\end{array}$ & $\begin{array}{r}57-43-23-25-57 \\
57-94-84-73-57 \\
57-96-57\end{array}$ & 224185 & 86.4 \\
\hline$S 31$ & 9 & 7 & 3000 & 3000 & $\begin{array}{r}57-58-49-29-17-57 \\
57-90-69-60-57 \\
57-88-99-57\end{array}$ & $\begin{array}{r}57-84-73-43-23-25-57 \\
57-96-94-57\end{array}$ & 236745 & 86.4 \\
\hline$S 32$ & 9 & 7 & 3000 & 2000 & $\begin{array}{l}57-49-29-17-57 \\
57-69-60-58-57 \\
57-88-99-90-57\end{array}$ & $\begin{array}{r}57-43-23-25-57 \\
57-94-84-73-57 \\
57-96-57\end{array}$ & 270259 & 86.7 \\
\hline
\end{tabular}


Table 4. Results of optimization of the research problem using BBO algorithm

\begin{tabular}{|c|c|c|c|c|c|c|c|c|}
\hline \multirow{2}{*}{ 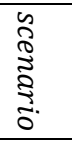 } & \multirow[b]{2}{*}{$N_{p}$} & \multirow[b]{2}{*}{$N_{d}$} & \multirow[b]{2}{*}{$Q_{p}$} & \multirow[b]{2}{*}{$Q_{d}$} & \multicolumn{4}{|c|}{$B B O$} \\
\hline & & & & & Tour $r_{\text {supplier }}$ & Tour $_{\text {customer }}$ & $f$ & $C P U(S)$ \\
\hline$S 33$ & 8 & 10 & 3500 & 3000 & $\begin{array}{r}57-60-49-29-17-57 \\
57-99-90-69-57 \\
57-88-57\end{array}$ & $\begin{array}{r}57-43-23-25-57 \\
57-64-52-55-57 \\
57-96-94-84-73-57\end{array}$ & 346214 & 106.9 \\
\hline$S 34$ & 8 & 10 & 3500 & 2000 & $\begin{array}{r}57-60-49-29-17-57 \\
57-99-90-69-57 \\
57-88-57\end{array}$ & $\begin{array}{r}57-43-23-25-57 \\
57-64-52-55-57 \\
57-84-73-57 \\
57-96-94-57\end{array}$ & 377996 & 106.8 \\
\hline$S 35$ & 8 & 10 & 3000 & 3000 & $\begin{array}{r}57-49-29-17-57 \\
57-69-90-60-57 \\
57-88-99-57\end{array}$ & $\begin{array}{r}57-43-23-25-57 \\
57-64-52-55-57 \\
57-96-94-84-73-57\end{array}$ & 395030 & 107.0 \\
\hline$S 36$ & 8 & 10 & 3000 & 2000 & $\begin{array}{r}57-49-29-17-57 \\
57-69-90-60-57 \\
57-88-99-57\end{array}$ & $\begin{array}{r}57-43-23-25-57 \\
57-64-52-55-57 \\
57-84-73-57 \\
57-96-94-57\end{array}$ & 435191 & 106.7 \\
\hline$S 37$ & 8 & 9 & 3500 & 3000 & $\begin{array}{r}57-60-49-29-17-57 \\
57-99-90-69-57 \\
57-88-57\end{array}$ & $\begin{array}{r}57-55-43-23-25-57 \\
57-96-94-84-73-64-57\end{array}$ & 293494 & 91.0 \\
\hline$S 38$ & 8 & 9 & 3500 & 2000 & $\begin{array}{r}57-60-49-29-17-57 \\
57-99-90-69-57 \\
57-88-57\end{array}$ & $\begin{array}{r}57-43-23-25-57 \\
57-84-73-64-55-57 \\
57-96-94-57\end{array}$ & 303296 & 90.9 \\
\hline$S 39$ & 8 & 9 & 3000 & 3000 & $\begin{array}{r}57-49-29-17-57 \\
57-69-90-60-57 \\
57-88-99-57\end{array}$ & $\begin{array}{r}57-55-43-23-25-57 \\
57-96-94-84-73-64-57\end{array}$ & 319793 & 90.8 \\
\hline$S 40$ & 8 & 9 & 3000 & 2000 & $\begin{array}{r}57-49-29-17-57 \\
57-69-90-60-57 \\
57-88-99-57\end{array}$ & $\begin{array}{r}57-43-23-25-57 \\
57-84-73-64-55-57 \\
57-96-94-57\end{array}$ & 345853 & 90.9 \\
\hline$S 41$ & 8 & 8 & 3500 & 3000 & $\begin{array}{r}57-60-49-29-17-57 \\
57-99-90-69-57 \\
57-88-57\end{array}$ & $\begin{array}{r}-64-73-43-23-25-57 \\
57-84 \\
57-96-94-57\end{array}$ & 202983 & 85.9 \\
\hline$S 42$ & 8 & 8 & 3500 & 2000 & $\begin{array}{r}57-60-49-29-17-57 \\
57-99-90-69-57 \\
57-88-57\end{array}$ & $\begin{array}{r}57-43-23-25-57 \\
57-84-73-64-57 \\
57-96-94-57\end{array}$ & 218544 & 86.1 \\
\hline$S 43$ & 8 & 8 & 3000 & 3000 & $\begin{array}{r}57-49-29-17-57 \\
57-69-90-60-57 \\
57-88-99-57\end{array}$ & $\begin{array}{r}-64-73-43-23-25-57 \\
57-84 \\
57-96-94-57\end{array}$ & 251663 & 86.2 \\
\hline$S 44$ & 8 & 8 & 3000 & 2000 & $\begin{array}{r}57-49-29-17-57 \\
57-69-90-60-57 \\
57-88-99-57\end{array}$ & $\begin{array}{r}57-43-23-25-57 \\
57-84-73-64-57 \\
57-96-94-57\end{array}$ & 259433 & 86.1 \\
\hline
\end{tabular}


Table 5. Results of optimization of the research problem using BBO algorithm

\begin{tabular}{|c|c|c|c|c|c|c|c|c|}
\hline \multirow{2}{*}{ 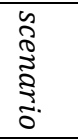 } & \multirow[b]{2}{*}{$N_{p}$} & \multirow[b]{2}{*}{$N_{d}$} & \multirow[b]{2}{*}{$Q_{p}$} & \multirow[b]{2}{*}{$Q_{d}$} & \multicolumn{4}{|c|}{ GAMS } \\
\hline & & & & & Tour $_{\text {supplier }}$ & Tour $_{\text {customer }}$ & $f$ & $C P U(S)$ \\
\hline$S 45$ & 8 & 7 & 3500 & 3000 & $\begin{array}{r}57-60-49-29-17-57 \\
57-99-90-69-57 \\
57-88-57\end{array}$ & $\begin{array}{r}57-84-73-43-23-25-57 \\
57-96-94-57\end{array}$ & 184556 & 72.5 \\
\hline$S 46$ & 8 & 7 & 3500 & 2000 & $\begin{array}{r}57-60-49-29-17-57 \\
57-99-90-69-57 \\
57-88-57\end{array}$ & $\begin{array}{r}57-43-23-25-57 \\
57-94-84-73-57 \\
57-96-57\end{array}$ & 198213 & 72.6 \\
\hline$S 47$ & 8 & 7 & 3000 & 3000 & $\begin{array}{r}57-49-29-17-57 \\
57-69-90-60-57 \\
57-88-99-57\end{array}$ & $\begin{array}{r}57-84-73-43-23-25-57 \\
57-96-94-57\end{array}$ & 210782 & 72.0 \\
\hline$S 48$ & 8 & 7 & 3000 & 2000 & $\begin{array}{r}57-49-29-17-57 \\
57-69-90-60-57 \\
57-88-99-57\end{array}$ & $\begin{array}{r}57-43-23-25-57 \\
57-94-84-73-57 \\
57-96-57\end{array}$ & 227853 & 72.9 \\
\hline$S 49$ & 7 & 10 & 3500 & 3000 & $\begin{array}{r}57-60-49-29-17-57 \\
57-99-90-69-57\end{array}$ & $\begin{array}{r}57-43-23-25-57 \\
57-64-52-55-57 \\
57-96-94-84-73-57 \\
\end{array}$ & 266826 & 97.6 \\
\hline$S 50$ & 7 & 10 & 3500 & 2000 & $\begin{array}{r}57-60-49-29-17-57 \\
57-99-90-69-57\end{array}$ & $\begin{array}{r}57-43-23-25-57 \\
57-64-52-55-57 \\
57-84-73-57 \\
57-96-94-57\end{array}$ & 279394 & 98.0 \\
\hline$S 51$ & 7 & 10 & 3000 & 3000 & $\begin{array}{r}57-60-49-29-17-57 \\
57-90-69-57 \\
57-99-57\end{array}$ & $\begin{array}{r}57-43-23-25-57 \\
57-64-52-55-57 \\
57-96-94-84-73-57\end{array}$ & 287879 & 97.9 \\
\hline$S 52$ & 7 & 10 & 3000 & 2000 & $\begin{array}{r}57-60-49-29-17-57 \\
57-90-69-57 \\
57-99-57\end{array}$ & $\begin{array}{r}57-43-23-25-57 \\
57-64-52-55-57 \\
57-84-73-57 \\
57-96-94-57\end{array}$ & 302047 & 98.0 \\
\hline$S 53$ & 7 & 9 & 3500 & 3000 & $\begin{array}{r}57-60-49-29-17-57 \\
57-99-90-69-57\end{array}$ & $\begin{array}{r}57-55-43-23-25-57 \\
57-96-94-84-73-64-57\end{array}$ & 217871 & 86.5 \\
\hline$S 54$ & 7 & 9 & 3500 & 2000 & $\begin{array}{r}57-60-49-29-17-57 \\
57-99-90-69-57\end{array}$ & $\begin{array}{r}57-43-23-25-57 \\
57-84-73-64-55-57 \\
57-96-94-57\end{array}$ & 239658 & 86.6 \\
\hline$S 55$ & 7 & 9 & 3000 & 3000 & $\begin{array}{r}57-60-49-29-17-57 \\
57-90-69-57 \\
57-99-57\end{array}$ & $\begin{array}{r}57-55-43-23-25-57 \\
57-96-94-84-73-64-57\end{array}$ & 254909 & 86.4 \\
\hline$S 56$ & 7 & 9 & 3000 & 2000 & $\begin{array}{r}57-60-49-29-17-57 \\
57-90-69-57 \\
57-99-57\end{array}$ & $\begin{array}{r}57-43-23-25-57 \\
57-84-73-64-55-57 \\
57-96-94-57\end{array}$ & 276696 & 86.0 \\
\hline
\end{tabular}


Table 6. Results of optimization of the research problem using BBO algorithm (continued)

\begin{tabular}{|c|c|c|c|c|c|c|c|c|c|c|c|c|c|}
\hline \multirow{2}{*}{ 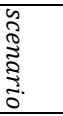 } & \multirow[b]{2}{*}{$N_{p}$} & \multirow[b]{2}{*}{$N_{d}$} & \multirow[b]{2}{*}{$Q_{p}$} & \multirow[b]{2}{*}{$Q_{d}$} & \multicolumn{4}{|c|}{$B B O$} & \multicolumn{4}{|c|}{ GAMS } & \multirow[b]{2}{*}{ Gap $\%$} \\
\hline & & & & & Tour $_{\text {supplier }}$ & Tour $_{\text {customer }}$ & $f$ & $C P U(S)$ & Tour $_{\text {supplier }}$ & Tour $_{\text {customer }}$ & $f$ & $C P U(S)$ & \\
\hline$S 57$ & 7 & 8 & $\begin{array}{c}350 \\
0\end{array}$ & $\begin{array}{c}300 \\
0\end{array}$ & $\begin{array}{c}-60-49-29-17-57 \\
57 \\
57-99-90-69-57\end{array}$ & $\begin{array}{c}-73-43-23-25-57 \\
57-84-64 \\
57-96-94-57\end{array}$ & 186737 & 113.5 & - & - & - & - & - \\
\hline$S 58$ & 7 & 8 & $\begin{array}{c}350 \\
0\end{array}$ & $\begin{array}{c}200 \\
0\end{array}$ & $\begin{array}{c}-60-49-29-17-57 \\
57 \\
57-99-90-69-57\end{array}$ & $\begin{array}{c}57-43-23-25-57 \\
57-84-73-64-57 \\
57-96-94-57\end{array}$ & 206867 & 112.5 & - & - & - & - & - \\
\hline$S 59$ & 7 & 8 & $\begin{array}{c}300 \\
0\end{array}$ & $\begin{array}{c}300 \\
0\end{array}$ & $\begin{array}{c}-60-49-29-17-57 \\
57 \\
57-90-69-57 \\
57-99-57\end{array}$ & $\begin{array}{c}-73-43-23-25-57 \\
57-84-64 \\
57-96-94-57\end{array}$ & 216055 & 116.7 & - & - & - & - & - \\
\hline$S 60$ & 7 & 8 & $\begin{array}{c}300 \\
0\end{array}$ & $\begin{array}{c}200 \\
0\end{array}$ & $\begin{array}{c}-60-49-29-17-57 \\
57 \\
57-90-69-57 \\
57-99-57\end{array}$ & $\begin{array}{c}57-43-23-25-57 \\
57-84-73-64-57 \\
57-96-94-57\end{array}$ & 220536 & 115.8 & - & - & - & - & - \\
\hline$S 61$ & 7 & 7 & $\begin{array}{c}350 \\
0\end{array}$ & $\begin{array}{c}300 \\
0\end{array}$ & $\begin{array}{c}-60-20-28-27-57 \\
57 \\
57-99-90-70-57\end{array}$ & $\begin{array}{c}-84-73-33-25-57 \\
57-66 \\
57-86-94-57\end{array}$ & 164460 & 82.0 & $\begin{array}{c}-60-49-29-17-57 \\
57 \\
57-99-90-69-57\end{array}$ & $\begin{array}{c}-43-23-25-57 \\
57-84-73 \\
57-96-94-57\end{array}$ & 162748 & 4267 & 1.052 \\
\hline$S 62$ & 7 & 7 & $\begin{array}{c}350 \\
0\end{array}$ & $\begin{array}{c}200 \\
0\end{array}$ & $\begin{array}{c}-60-49-29-17-57 \\
57 \\
57-99-90-69-57\end{array}$ & $\begin{array}{c}57-43-23-25-57 \\
57-94-84-73-57 \\
57-96-57\end{array}$ & 172041 & 82.1 & $\begin{array}{c}-60-49-29-17-57 \\
57 \\
57-99-90-69-57\end{array}$ & $\begin{array}{c}-43-23-25-57 \\
57 \\
-94-84-73-57 \\
57 \\
57-96-57 \\
\end{array}$ & 172041 & 4316 & 0 \\
\hline$S 63$ & 7 & 7 & $\begin{array}{c}300 \\
0\end{array}$ & $\begin{array}{c}300 \\
0\end{array}$ & $\begin{array}{c}-60-49-29-17-57 \\
57 \\
57-90-69-57 \\
57-99-57\end{array}$ & $\begin{array}{c}-73-43-23-25-57 \\
57-84 \\
57-96-94-57\end{array}$ & 179853 & 82.1 & $\begin{array}{c}-60-49-29-17-57 \\
57 \\
57-90-69-57 \\
57-99-57\end{array}$ & $\begin{array}{c}-43-23-25-57 \\
57-84-73 \\
57-96-94-57\end{array}$ & 179853 & 4749 & 0 \\
\hline$S 64$ & 7 & 7 & $\begin{array}{c}300 \\
0\end{array}$ & $\begin{array}{c}200 \\
0\end{array}$ & $\begin{array}{c}-60-40-19-7-57 \\
57 \\
57-70-57 \\
57-89-90-57 \\
57-96-99-57 \\
\end{array}$ & $\begin{array}{c}57-42-14-26-57 \\
57-81-63-57 \\
57-94-73-57 \\
57-96-95-57\end{array}$ & 207351 & 82.0 & $\begin{array}{c}-60-49-29-17-57 \\
57 \\
57-90-69-57 \\
57-99-57\end{array}$ & $\begin{array}{c}-43-23-25-57 \\
57 \\
-94-84-73-57 \\
57 \\
57-96-57\end{array}$ & 202947 & 4913 & 2.17 \\
\hline
\end{tabular}

\section{The results of Table (1) showed that:}

1. With decrease in the pickup vehicle capacity, total pickup costs increased. This was because the sub-tours created on the product pickup routes from the supplier side was subject to performance capacity limitations, leading to the need for more sub-tours, resulting in an increase in the total cost of the system. This was also true for reducing the capacity of vehicles for the type of delivery. In this condition, vehicles that have to supply customers had a capacity limit, resulting in higher system costs.

2. The increase in the number of customers and the number of suppliers had a direct effect on the increase in the total cost of the system, as more paths were needed to supply the demand and deliver the order to customers. Additionally, more time should be taken when picking up the product from the supply chain. This is seen in Figures (3) to (6).

3. The maximum difference between the results from GAMS software and BBO algorithm was $2.17 \%$, showing that the efficiency of the proposed solving algorithm was proper. Moreover, compared with the solving time, BBO algorithm was superior to GAMS as in the case of solving scenarios from 61 to 64 for a suggested solving method in about 82 seconds. This value was more than 4000 seconds in GAMS algorithm.

4. With increase in the capacity of pick-up and delivery vehicles, greater sub-tours were created. 


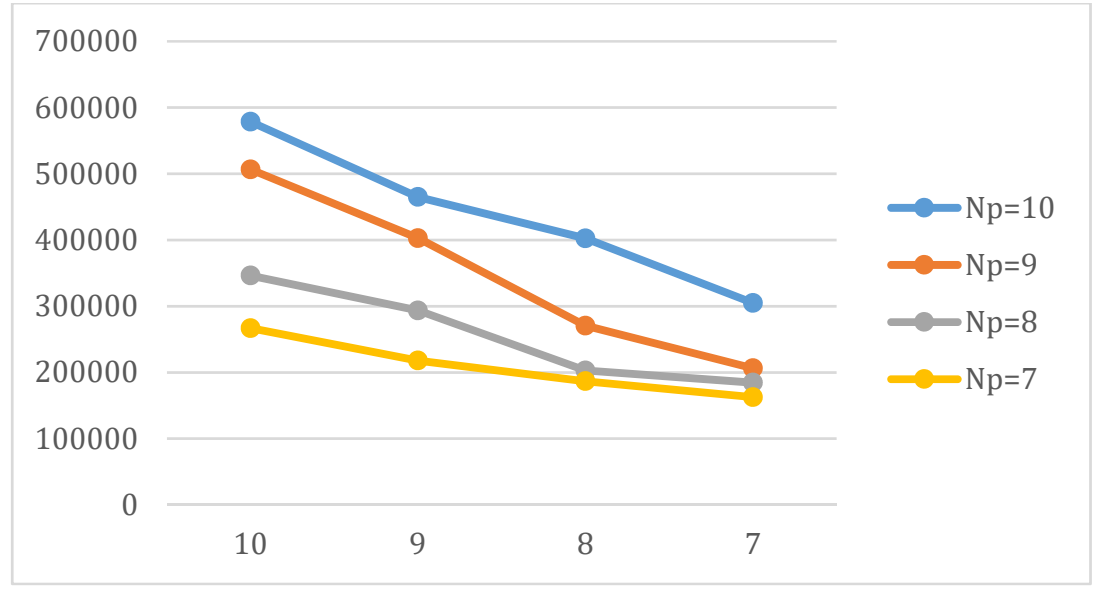

Figure 3. Changes in the value of objective function against changes in Np at a time $Q p=3500$ and $Q d=3000$

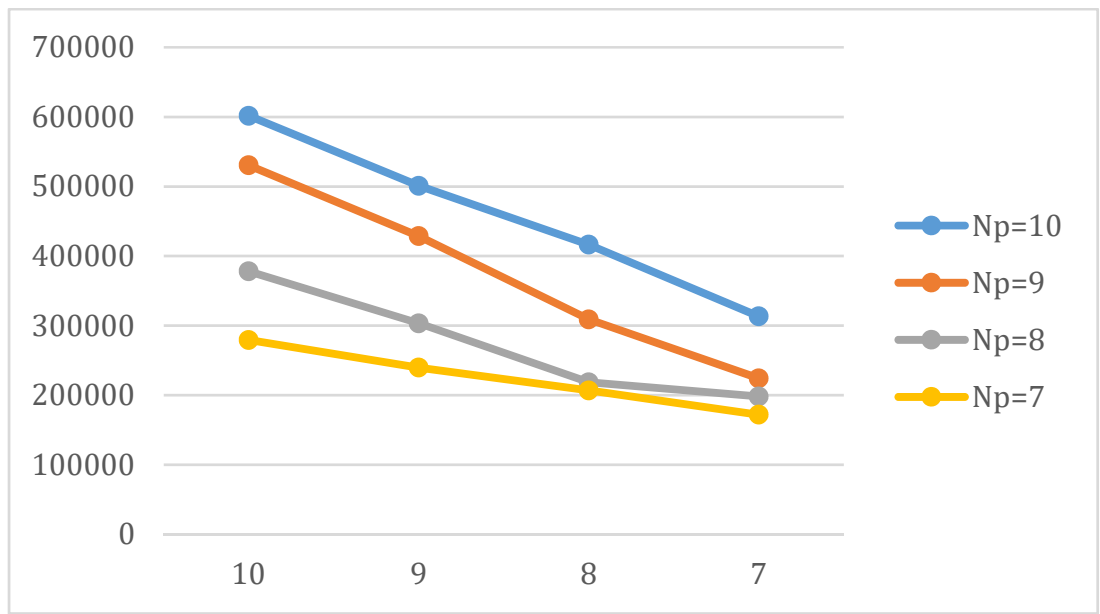

Figure 4. Changes in the value of objective function against changes of $\mathrm{Np}$ at time $\mathrm{Qp}=3500$ and $\mathrm{Qd}=2000$

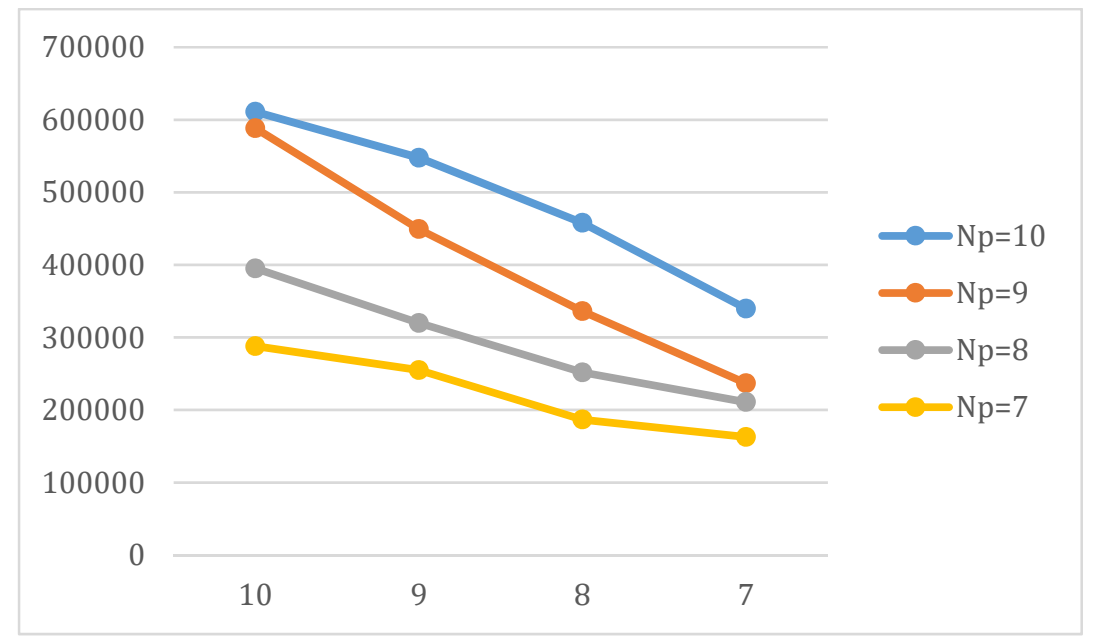

Figure 5. Changes in the value of objective function against changes in Np at times $\mathrm{Qp}=3000$ and $\mathrm{Qd}=3000$ 


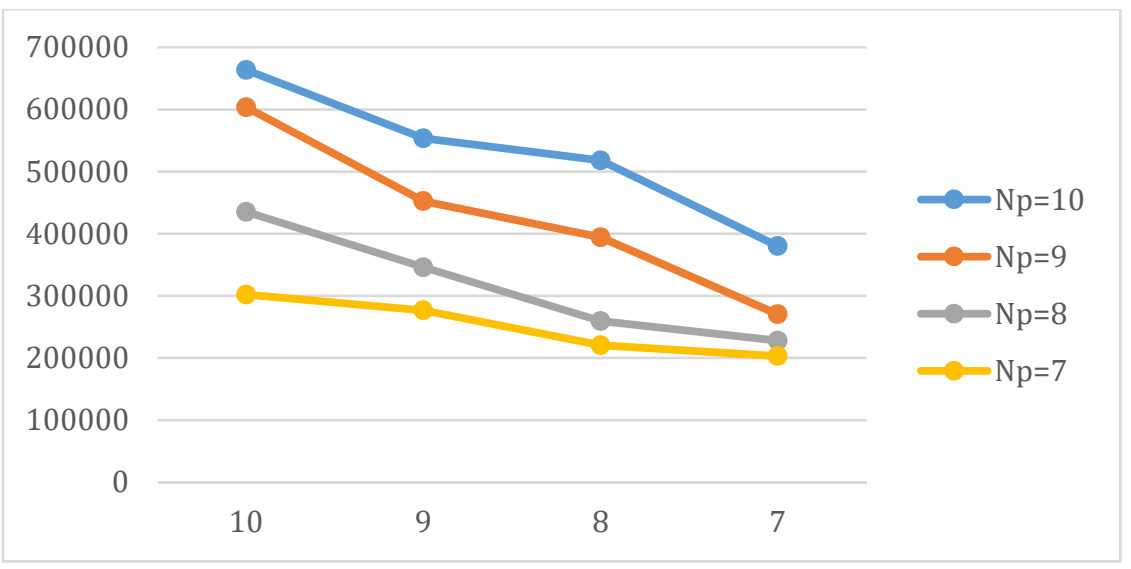

Figure 6. Changes in the value of objective function against changes in Np at times $\mathrm{Qp}=3000$ and $\mathrm{Qd}=2000$

\section{Comparison of the proposed solution method (BBO) with PSO}

In this section, to examine the function of the proposed solution method further, the results have been compared with the developed PSO algorithm as developed by Chen et al. (2016). In doing so, the results of scenarios 61 to 64 were analyzed using PSO and BBO algorithms. Table 4 shows the results of this comparison.

According to the results of Table 4-2 and concerning the limitations of the software and the time limitations, (5,000 seconds), only 4 of the last scenarios were all comparable solutions that can be compared after the comparison in S61 and S62 scenarios, the proposed behavioral algorithm was better than PSO algorithm written in Chen et al. (2016). In S63 scenario, both solutions have reached the optimal answer not superior to each other. In S64 scenario, the performance of PSO algorithm was better than BBO. Thus, it was concluded that the overall performance of BBO algorithm was better than PSO algorithm. In the next section, using BBO algorithm, the sensitivity of $\alpha$ value have been analyzed.

\section{- Analysis and sensitivity analysis at the confidence level (1- $\alpha)$}

In this section, the sensitivity of $\alpha$ surface was analyzed on S61 scenario. It should be noted that the level $\alpha=0.1$ was used in the scenarios described in sections (2) and (3).

Table 3. Sensitivity analysis at $\alpha$ level

\begin{tabular}{|c|c|c|c|c|c|c|c|c|c|}
\hline$\alpha$ & 0.01 & 0.025 & 0.05 & 0.075 & 0.10 & 0.125 & 0.15 & 0.175 & 0.20 \\
\hline$f$ & 170966 & 168489 & 164460 & 162389 & 152873 & 148986 & 144924 & 133576 & 107489 \\
\hline
\end{tabular}

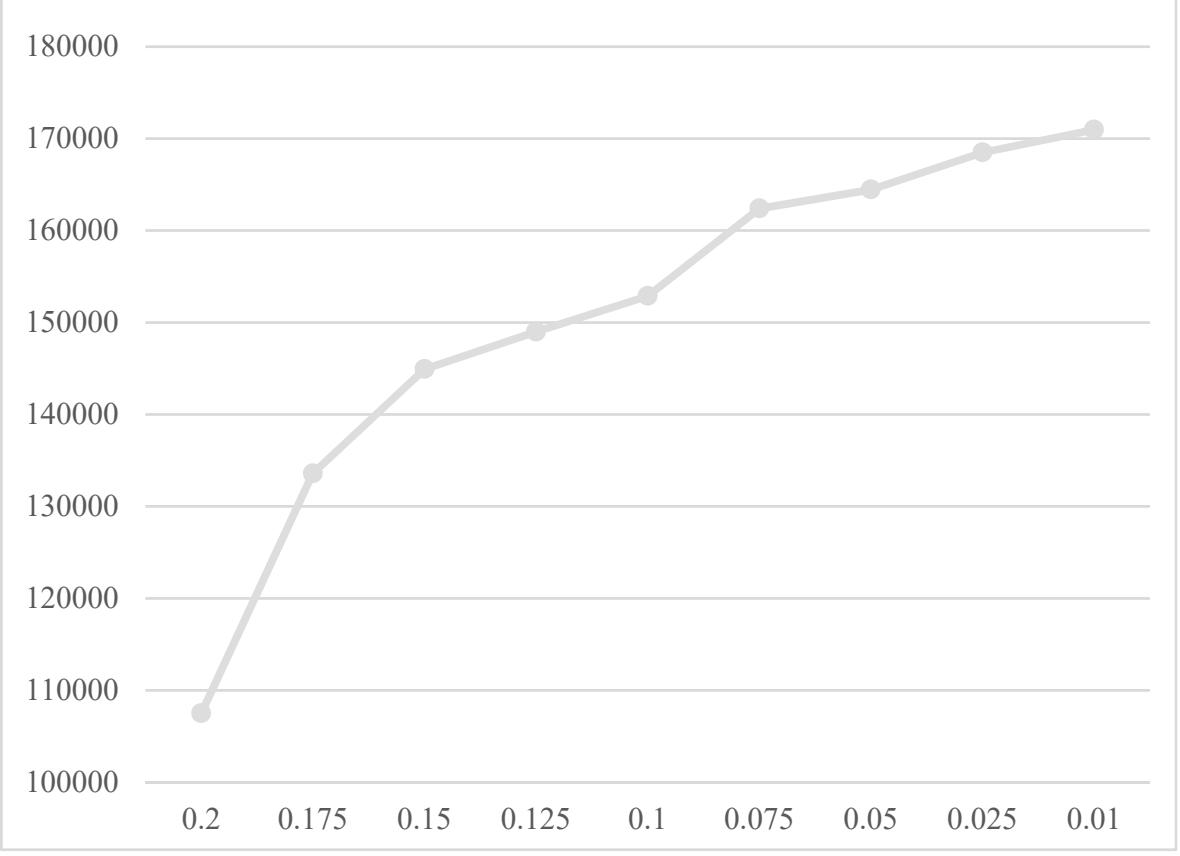

Figure 7. Results of sensitivity analysis at the surface $\alpha$ 
According to the results of Table (3) and Figure (7), one can see that as the value of $\alpha$ increased, the total cost of transportation grew. This is because the high transportation time would be higher for access points in the networks of the transportation network, which would result in increased transportation costs.

\section{Conclusion}

\section{The main research question}

Optimal policies for receiving, sorting and labeling of multiple products for cross-dock as well as optimal routes for vehicles with regard to non-deterministic motion times at two levels: How are 1) supply points and crossdocks; and 2) cross-docks and delivery points?

In this study, a collecting, sorting and sending flows system for a set of suppliers and customers was considered in form of multiple cross-dock problem where the pickup and sending vehicle moving times were not predetermined value and followed a normal distribution. Moreover, given the existence of maximum time allowed to deliver the goods to customers, the delayed or sooner delivery and pickup should be controlled not to dissatisfy the customer. The present study tried to examine and optimize the combination of vehicle routing in uncertainty situations at delivery and withdrawal times from cross-dock shipments to meet customer satisfaction and the total cost of sending and receiving minimum demand.

\section{Sub-questions}

1. How is the allocation of pick-up vehicles to the cross-docks?

Table (1) showed how the vehicles were allocated to cross-docks. Accordingly, the suppliers have been installed in routes that, while reducing the number of existing sub-tours, had a small distance from the cross-docks.

\section{How are vehicles at the delivery points allocated to cross-docks?}

Customer centers were connected to cross-docks to form a balanced flow in each sub-tour. In other words, we do not see a large number of customers in a sub-tour, as opposed to a small number of customers in other domains. This is seen in Table (3).

\section{How are the optimal sub-tours drivers for vehicles moving at the level of supply points and cross- docks?}

With reduction in the capacity of the pickup vehicles, total costs increased. This is because the sub-tours created on the pickup routes from the supplier side was subject to capacity limitations, ending in the need for more subtours, resulting in an increase in the total cost of the system.

\section{How to determine the optimum sub-tours of the vehicles movement at cross-docks and customer centers?}

The results showed that the increase in the capacity of the vehicles, delivery of the sub-tours was made up of larger tours. This was examined in 64 optimized scenarios. Additionally, the results indicated that the maximum difference between the solution obtained from GAMS software and BBO algorithm was $2.17 \%$, showing that the efficiency of the proposed solving algorithm was proper.

\section{References}

[1] Dehbari, S., Pour Rousta, A.R., Naderi Bani, M., Ghobadian, E., Tavakoli Moghadam, R. (2012). Routing multi-purpose vehicles with possible service time and fuzzy demand under time window constraints, Operations Research Journal Applications, No. 4

[2] Jafari, A., Chiniforoushan, P., Sadeghi Sarvestani, (2011). Tricia Refrigeration Simulation Algorithm for Solving the Multi-Storm Vehicle Routing Problem, The First National Conference on Knowledge of Computer and Information Technology, Tabriz Publications, Computer Science Department.

[3] Apte UM, \& Viswanathan, S. (2000). Effective Cross Docking for Improving Distribution Efficiencies. International Journal of Logistics: Research and Applications. 3(3):291-302.

[4] Dantzig, G. B., Ramser, J. H. (1959) "The truck dispatching problem“, Management Science 6 (1), pp. 80-91.

[5] Barbucha ,D.2012. Search modes for the cooperative multi-agent system solving the vehicle routing problem, Neurocomputing Journal, Vol 88, PP.13-23

[6] Arnaout, J.-P, Musa, R.,. and Jung, H., "Ant colony optimization algorithm to solve for the transportation problem of cross-docking network", Computers \& Industrial Engineering, Vol. 59, No. 1, (2010), 85-92.

[7] Velazquez, M., David Claudio and A. Ravi Ravindran. (2010). Experiments in Multiple Criteria Selection Problems with Multiple Decision Makers. International Journal of Operational Research 7(4)

[8] Taş, D., Dellaert,N., Van Woensel,T and de Kok,T .2013. Vehicle routing problem with stochastic travel times including soft time windows and service costs, Computers \& Operations Research, Vol. 40, No. 1, 214-224.

[9] LiangjunKe, Zuren Feng .2013. A two-phase metaheuristic for the cumulative capacitated vehicle routing problem, Vol. 40, No. 2, 633-638.

[10] Goksal , F.P., Goksal I and Altiparmak,F.2013. A hybrid particle swarm optimization for vehicle routing problem with simultaneous pickup and delivery, Computers \& Industrial Engineering 65(1):39-53 DOI: 10.1016/j.cie.2012.01.005

[11] Xiao, Y., Tian,Y and Zhao,Q.2014. Optimizing frequent time-window selection for association rules mining in a temporal database using a variable neighbourhood search, Computers \& Operations Research, Vol. 52, No. 1, 241-250. 
[12] Briesemeister, R and Novaes Antônio G. N.,2017. Comparing an Approximate Queuing Approach with Simulation for the Solution of a Cross-Docking Problem, Journal of Applied Mathematics, Volume 2017 (2017), Article ID 4987127, 11 pages.

[13] Aasadi,Z., Mohammad Valipourkhatir,M and safaei, S. 2016, Modeling and solving Multi-objective Vehicle Routing Problem of Distribution Companies with Fuzzy and Stochastic Constraints, Journal of Modern Research in Decision Making, Volume 4, Issue 1, Page 1-24

[14] Madani-Isfahani, M., Tavakkoli-Moghaddam, R., and Naderi, B., "Multiple cross-docks scheduling using two meta-heuristic algorithms", Computers \& Industrial Engineering, Vol. 74, (2014), 129-138.

[15] Li, Q., Nie, Y., Vallamsundar, S., Lin, J. \&Homem-de-Mello, T. (2016). Finding Efficient and Environmentally Friendly Paths for Risk-Averse Freight Carriers. Netw Spat Econ, 16(1), 255- 275. 